Historic, Archive Document

Do not assume content reflects current scientific knowledge, policies, or practices. 



\section{2,57 FARM AND GARDEN SEEDS}

\section{Market Gardeners' Price List \\ * MAR 5 180. *

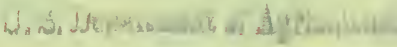 \\ TAYLOR BROTHERS}

$\nabla$

1930

CAMDEN, NEW JERSEY

IMPLEMENTS FOR THE FARM regetable Seed I prals D. N. Shoemaker FEB 211930

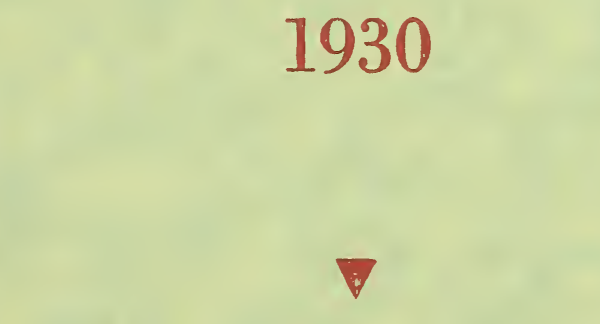





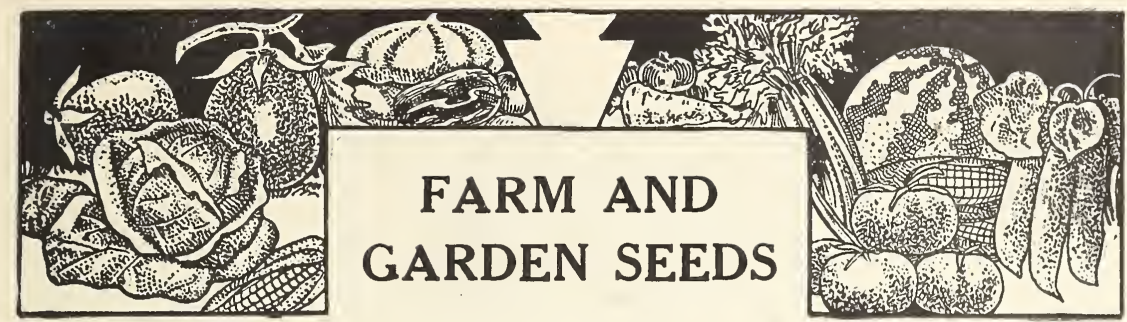

\section{CERTIFIED SEED POTATOES}

PRINCE EDWARD ISLAND AND MAINE GROWN, 10 peck sacks IRISH COBBLERS (good keepers, vigorous and popular)

GREEN MOUNTAINS (big cropper, splendid cooker).-Market Prices

\section{ONION SETS-Extra Fancy}

JAPANESE

Bus.

$1 / 2$ Bus.

YELLOW

3.75

$\$ 2.10$

$1 \mathrm{Lb}$.

WHITE.

4.25

2.00

2.25

$\$ 0.20$

32 lbs. per bushel. Bags extra

\section{SHEEP'S HEAD SHEEP MANURE}

5 lbs. . $\$ 0.35$

25 lbs. . $\$ 1.00$

2000 lbs. . $\$ 42.00$

$10 \mathrm{lbs}$. . .50

$50 \mathrm{lbs} . .1 .55$

1000 lbs. . 43.00 ton

500 lbs. . $\$ 44.00$ ton

$100 \mathrm{lbs}$. $2.50 \mathrm{cwt}$.

\section{MAKE MORE MONEY with BUCKEYE INCUBATORS AND BROODERS}

\section{Efficient, Economical, Easy of Operation STANDARD INCUBATORS}

No. 2. 175 eggs $\ldots \ldots . \$ 35.00 \quad$ No. 3. 250 eggs $\ldots \ldots \ldots 46.50$ No.4. 350 eggs $\ldots \ldots .55 .00 \quad$ No. 5. 600 eggs $\ldots \ldots .87 .50$ All equipped with automatic turning trays

\section{BUCKEYE COAL BURNING BROODERS}

No. 117. 350 chicks......\$14.75 No. 118. 500 chicks......\$18.50 No. 119.1000 chicks..... $\$ 22.50$ Freight allowed

\section{STAR GARDEN WHEELBARROWS}

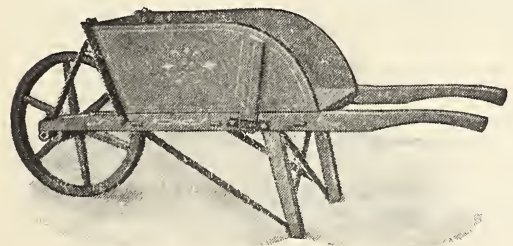

Guaranteed to be the best barrow made.

$23 / 4$ inch tread, steel axle, wood hub, wood spokes and rim. Bright red, No. 3, $\$ 6.50$ each; No. $4, \$ 5.00$ each. 


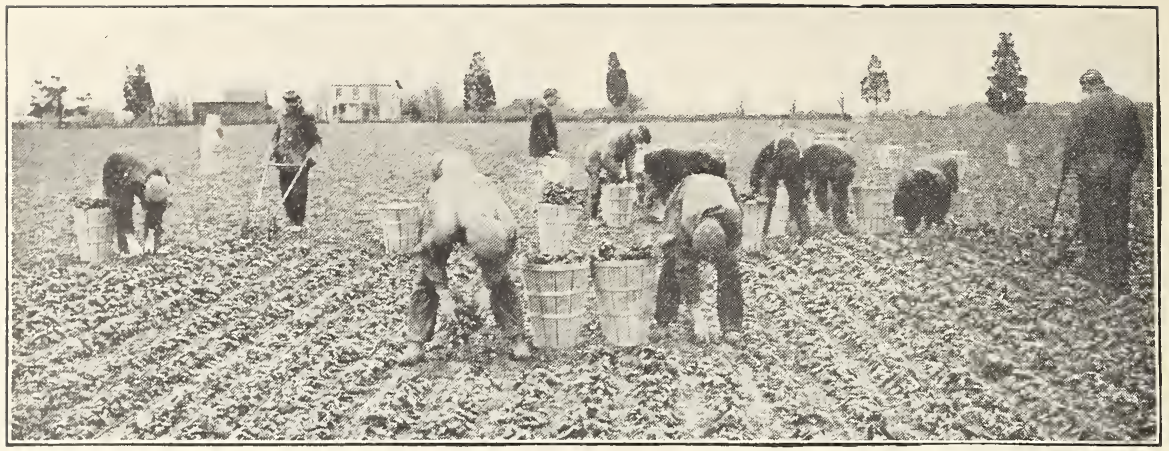

Scene of a South Jersey Spinach Field

\section{FARM AND GARDEN SEEDS}

W E ARE STRICTLY a garden, farmers' seed and implement supply house, using the same care in selection of our seed stock from reliable growers as would be exercised by the most particular gardener or trucker.

While we believe prices quoted are the best prices to be offered, still they are subject to market changes.

SPECIAL-DON'T FORGET-When ordering seed sent by mail to include postage.

\section{PEAS-Extra Early}

$1 \mathrm{lb} .15$ lbs. $100 \mathrm{lbs}$.

Alaska, 2 ft. $\$ 0.26 \$ 2.55 \$ 16.00$ * American Wonder, $1 \mathrm{ft}, \ldots \quad .26 \quad 2.55 \quad 16.00$ Alaska, large pod, $2 \mathrm{ft} . \ldots \ldots \ldots . .26 \quad 2.55 \quad 16.00$ First and Best, $21 / 2$ ft............. *Gradue or Prosperity, 3 ft. . $\quad .27 \quad 2.70 \quad 17.00$ $\begin{array}{lllll} & \text { Thomas Laxton, } 3 \mathrm{ft} \ldots \ldots \ldots & .28 & 2.85 & 18.00\end{array}$ Premium Gems, $11 \frac{1}{2}$ ft. . . . . . .26 $2.55 \quad 16.00$

\section{PEAS-Second Early Main Crop}

1 lb. 15 lbs. 100 lbs.

Laxtonian $\$ 0.28 \quad \$ 2.85 \quad \$ 18.00$ Little Marvel. $\begin{array}{lll}.26 & 2.55 & 16.00\end{array}$

$\begin{array}{llll}\text { Market Garden Horsford, } 2 \text { ft. } & .26 & 2.55 & 16.00\end{array}$ *Stratagem "Improved," $2 \mathrm{ft}$. $\quad .28 \quad 2.85 \quad 18.00$

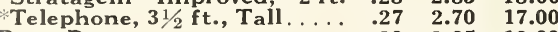
Peter Pan

Luscious Sugar.

World's Record

23.00

Wrinkled variety Peas marked thus* FIELD PEAS 15 lbs. bu. 100 lbs.
1.00
3.60

Canada COW PEAS

Gray Crowders.

New Era.

Whippoorwill Brown

Bus. $\$ 6.00$ 6.00 6.00

\section{SOJA BEANS}

Mammoth Yellow

Wilson Black.

\section{ASPARAGUS}

Barr's Mammoth

Palmetto.

Giant Argenteuil \$ 15 lb. $\begin{array}{rr}.15 & \$ 0.30 \\ .15 & .30\end{array}$

Washington Pedigreed

$.15 \quad .30$

Bus. Write for Prices

\section{ASPARAGUS ROOTS}

Barr's Mammoth (2 year old). Conover Colossal (2 year old)

Donald's Elmira ( 2 year old) Giant Argenteuil (2 year old) per 100 or 1000 Palmetto (2 year old)

$$
\begin{aligned}
& \text { Write } \\
& \text { for } \\
& \text { Prices }
\end{aligned}
$$

One year old, less $5 \mathrm{c}$ per 100 and $50 \mathrm{c}$ per 1000

\section{BEET-Turnip Rooted Variety}

Detroit Dark Red ...........\$0.15 $\$ 0.35 \$ \$ 1.00$ oz. $1 / 4$ lb. Early Eclipse Red Turnip.... $\quad .15 \quad .35 \quad 1.00$ EgyptianBloodTurnipCrosbys $\quad .15 \quad .35 \quad 1.00$ Extra Early Bassano Turnip.. $.15 \quad .35 \quad 1.00$ Early Blood Turnip......... $.15 \quad .35 \quad 1.00$ Lentz Extra Early Red Turnip $\begin{array}{llll}.15 & .35 & 1.00\end{array}$

\section{BEET-Mangel Wurzel}

Carter's Mammoth Long Red oz. 1/4 lib. lb.

Imperial Sugar Lane's.......

Swiss Chard.............

\section{BEANS-Green Pod Bush}

1 lb. 15 lbs. 100 lbs.

Bountiful, Stringless Flat Pod $\quad .39 \quad 4.50 \quad 29.00$ Longfellow, Early Round, $\begin{array}{lllll}\text { Prolific.................. } 32 & 3.45 & 22.00\end{array}$ Red Kidney ............... $31 \quad 3.30 \quad 21.00$ Refugee Early, Round Prolific $\begin{array}{llll}31 & 3.30 & 21.00\end{array}$ $\begin{array}{lllll}\text { Refugee Late } \ldots \ldots \ldots \ldots \ldots \ldots & .32 & \mathbf{3 . 4 5} & 22.00\end{array}$ Stringless Giant Pod . . . . . . . . . Stringless Burpee's ................ Valentine Black........... $.32 \quad 3.45 \quad 22.00$ $\begin{array}{lllll}\text { Sure Crop Stringless . . . . . . . . } & .34 & \mathbf{3 . 7 5} & \mathbf{2 4 . 0 0}\end{array}$

\section{BEANS-Wax Pod Bush}

1 lb. 15 lbs. 100 lbs. Currie's Rust Proof ......... $\quad .33 \quad 3.60 \quad 23.00$ Golden Wax, Improved....... Pencil Pod Wax................33 $3.60 \quad 23.00$

\section{BEANS-Pole or Runners}

$1 \mathrm{lb} .15$ lbs. $100 \mathrm{lbs}$. Kentucky Wonder.............30 $3.30 \quad 21.00$

\section{BEANS-Bush Lima}

1 lb. 15 lbs. 100 lbs. Fordhook Bush.......... $.43 \quad 5.10 \quad 33.00$ Burpee's Improved Bush.....

\section{BEANS-Pole Lima}

1 lb. 15 lbs. 100 lbs.

Challenger or Dreer's Improved................ $40 \quad 40 \quad 4.15 \quad 30.00$

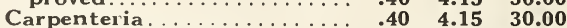
Jersey Extra Early . . . . . . . . . . King of the Garden.......... 


\section{BRUSSELS SPROUTS}

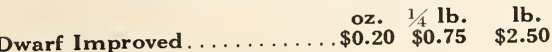

\section{BROCCOLI}

Purple Cape

$\begin{array}{lll}\text { oz. } & 1 / 4 \text { lb. } & \text { lb. } \\ .50 & 1.25 & 4.50\end{array}$

\section{CABBAGE-First Early}

\begin{tabular}{|c|c|}
\hline 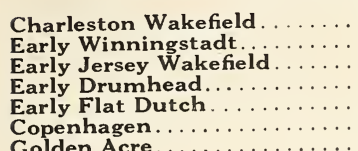 & $\begin{array}{l}.2 \\
.2 \\
.2 \\
.2 \\
.2 \\
.4 \\
.7\end{array}$ \\
\hline
\end{tabular}

\section{Second Early}

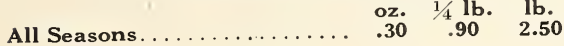

\section{Late Varieties}

\begin{tabular}{|c|c|c|}
\hline $\begin{array}{l}\text { Late Flat Dutch... } \\
\text { umhead Savoy. . . . . }\end{array}$ & $\begin{array}{l}\text { oz. } \\
.30 \\
.30\end{array}$ & $\begin{array}{c}1 / 4 \mathrm{lb} . \\
.90 \\
.75\end{array}$ \\
\hline $\begin{array}{l}\text { ium Large Late Drum- } \\
\text { d }{ }_{\text {h Ballhead } \ldots \ldots \ldots \ldots \ldots \ldots} \ldots \ldots \ldots \ldots \ldots \ldots\end{array}$ & $\begin{array}{l}.30 \\
.45\end{array}$ & $\begin{array}{r}.90 \\
1.40\end{array}$ \\
\hline
\end{tabular}

\section{CARROT}

Extra Early Forcing (10 days ahead of others) ........... Early Scarlet Horn (first early) Long White Belgian, for stock range Danvers, half-long Oxhart or Gerande........... Chantenay............. 15

Nantes ............... $\mathbf{. 1 5}$

Imp. Long Orange. . . . . . . . . .15 oz. $1 / 4$ lb. lb.

\section{CORN, FIELD CULTURE}

\section{Ensilage}

1 lb. bus.

Red Cob

\section{Yellow Dent Varieties}

1 pk. bus. 14 lbs. 56 lbs.

White Cap Yellow Dent

\section{Write}

Golden Beauty, 100 days

Improved Leaming, 90 days

for

Reids Yellow Dent.

for

\section{White Dent Varieties}

1 pk. bus.

14 lbs. 56 lbs.

Wisconsin White Dent

\section{SWEET OR SUGAR CORN}

\section{Extra Early Varieties}

1 lb. 15 lbs. 100 lbs.

Ferry New Early, Evergreen ... $\quad .29 \quad 2.60 \quad 16.00$ Hicknox Improved .......... $.30 \quad 2.70 \quad 17.00$

\section{Medium Early Varieties}

1 lb. 15 lbs. 100 lbs.

Golden Bantam

Golden Bantam
Howling Mob.

\section{Late Varieties}

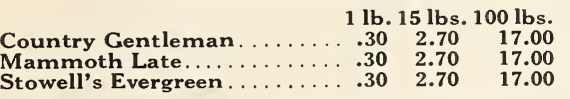

\section{CORN SALAD}

oz. $1 / 4$ lb. lb.

Broad Leaf $\$ 0.20 \$ 0.60 \$ 2.00$

\section{COLLARDS}

Georgia $\begin{array}{ccc}\text { oz. } & 1 / 4 \text { lb. } & \text { lb. } \\ .15 & .30 & .75\end{array}$

\section{CELERY}

Golden Self-Blanching oz. $1 / 4$ lb. lb.

$\begin{array}{lllll}\text { Golden Self-Blanching ........ } & .70 & 2.00 & 6.00 \\ \text { White Plume } & \ldots \ldots & .35 & 1.00 & 3.00\end{array}$

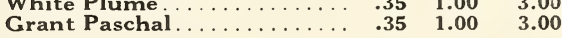

\section{CUCUMBER}

\begin{tabular}{|c|c|c|}
\hline 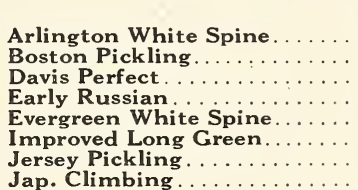 & $\begin{array}{l}\text { oz. } \\
.20 \\
.20 \\
.20 \\
.20 \\
.20 \\
.20 \\
.20 \\
.20\end{array}$ & $\begin{array}{c}1 / 4 \mathrm{lb} . \\
.45 \\
.45 \\
.50 \\
.45 \\
.45 \\
.50 \\
.45 \\
.45\end{array}$ \\
\hline
\end{tabular}

\section{ENDIVE}

Broad Leaf

Green Curled

oz. $1 / 4$ lb. lb.

$\begin{array}{rrr}.15 & .40 & 1.20 \\ .15 & .40 & 1.20\end{array}$

\section{KALE}

$\begin{array}{lccc} & \text { oz. } & 1 / 4 \text { lb. } & \text { lb. } \\ \text { Dwarf Green Curled Scotch . } & .15 & .35 & .95 \\ \text { Dwarf Siberian ... . . . . . . } & .15 & .35 & .95 \\ \text { Tall Green Curled Scotch.... } & .15 & .35 & .95\end{array}$

KOHL RABI

oz. $1 / 4$ lb. lb.

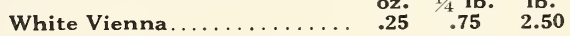

\section{LETTUCE}

\begin{tabular}{|c|c|c|}
\hline 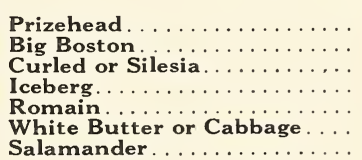 & $\begin{array}{l}0 z \\
.20 \\
.20 \\
.20 \\
.20 \\
.20 \\
.20 \\
.20\end{array}$ & $\begin{array}{l}1 / 4 b \\
.55 \\
.55 \\
.55 \\
.55 \\
.55 \\
.55 \\
.55\end{array}$ \\
\hline
\end{tabular}

Salamander............. $20 \quad .55 \quad 1.45$

\section{LEEK}

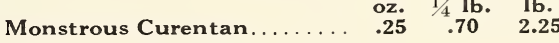

\section{MUSK MELON-Green Fleshed*}

\begin{tabular}{|c|c|c|c|}
\hline $\begin{array}{l}\text { *Extra Early Jennie Lind.... . . } \\
\text { *Extra Early Hackensack.... } \\
* \text { Early Netted Gem }\end{array}$ & $\begin{array}{l}\text { oz. } \\
.15 \\
.15\end{array}$ & $\begin{array}{c}1 / 4 \mathrm{lb} . \\
.40 \\
.40\end{array}$ & $\begin{array}{l}\text { lb. } \\
1.20 \\
1.20\end{array}$ \\
\hline Strain $\ldots \ldots \ldots \ldots \ldots$ & .15 & .40 & 1.20 \\
\hline ordhook .............. & .15 & .40 & 1.40 \\
\hline 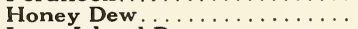 & .15 & .45 & 45 \\
\hline Island Beauty. & .15 & .40 & 1.20 \\
\hline $\operatorname{rd} \ldots \ldots \ldots$ & .1 & .40 & 1.10 \\
\hline d Gold Lined. & .15 & .40 & 1.20 \\
\hline
\end{tabular}

\section{MELON-Water}

\begin{tabular}{|c|c|c|}
\hline 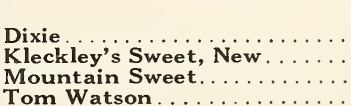 & $\begin{array}{l}\text { oz. } \\
.15 \\
.15 \\
.15 \\
.15\end{array}$ & $\begin{array}{c}1 / 4 \mathrm{~b} . \\
.30 \\
.40 \\
.40 \\
.30\end{array}$ \\
\hline
\end{tabular}

\section{NASTURTIUM}

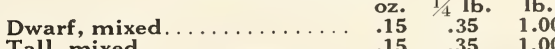

Tall, mixed........... 


\section{ONION}

Red Large Wethersfield . . . . . \$0.25 $\begin{array}{cc}0.21 / 4 & 16 . \\ \$ 0.65\end{array}$

Southport White Globe..... .25

Southport Yellow Globe..... . .25

South port Red Globe .......

White Portugal or Silver Skin $\quad .30$

Yellow Globe Danvers....... . .25

Yellow Prizetaker or Mammoth Spanish..........

$.25 \quad .60$

lb.

$\$ 2.35$

2.90

2.35

2.35

2.80

2.20

2.20

\section{OKRA}

Perkin's Perfected

oz. $1 / 4$ lb. lb.

\section{SQUASH-Winter Varieties}

\begin{tabular}{|c|c|}
\hline 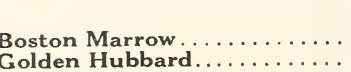 & $\begin{array}{l}\text { oz. } \\
.15 \\
.25\end{array}$ \\
\hline
\end{tabular}

\section{SQUASH-Summer Varieties}

Cocozelle....... Whish Scallop or oz. $1 / 4$ lb. lb. $.25 \quad .60$

1.50

Patty Pan...............

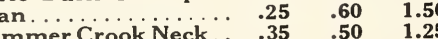

\section{PEPPER}

Large Bell, or Bull Nose... . . .

Long Red Cayenne...........

Ruby King

1.20

4.50

$.40 \quad 1.20$

4.75

4.50

\section{PARSLEY}

Champion Moss Curled......

Double Curled........... $\quad .15$

Plain or Single ............ $.15 \quad .35$

\section{PARSNIPS}

The Student Guernsey (extra large)

oz.

.15

.35

\section{PUMPKIN}

Connecticut Field.

Large Cashaw (striped) . . . . . . . . . . .

Quaker or Pie.

\section{RADISH}

Back Spanish (winter)...

China Rose (winter)

Chartier.

Early Long Scarlet (short top)

Early Scarlet Turnip (white

Early Scarlet French Break-$$
\text { fast }
$$

Icicle.

Philadelphia Box

Scarlet Globe.

\section{SPINACH}

Bloomsdale, Savoy, Leaved or oz. $1 / 4$ lb. lb. Norfolk..............\$0.10 \$0.20 \$0.45

\section{TOMATO}

\begin{tabular}{|c|c|c|}
\hline 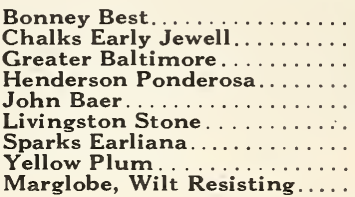 & $\begin{array}{l}.25 \\
.25 \\
.25 \\
.50 \\
.25 \\
.25 \\
.25 \\
.50 \\
.50\end{array}$ & $\begin{array}{r}.85 \\
.85 \\
.85 \\
1.60 \\
.85 \\
.85 \\
.85 \\
1.60 \\
1.60\end{array}$ \\
\hline
\end{tabular}

\section{TURNIP-Yellow Varieties}

American Grown

$\begin{array}{llll}15 & .25 & .75\end{array}$

Golden Ball ............. $15 \quad .25 \quad .75$

$\begin{array}{llll}\text { Purple-Top Yellow Rutabaga } & .15 & .25 & .75\end{array}$

White Rutabaga............

Yellow Aberdeen............. $\quad .15 \quad .25 \quad .75$

Yellow Globe . . . . . . . . . . . $\quad .15 \quad \begin{array}{lll}.25 & .75\end{array}$

\section{TURNIP - White Varieties}

American Grown

Early White Flat Dutch $15 \quad .25 \quad .75$

(strap-leaved)

Early Red-Top Globe........

$\begin{array}{llll}\text { Early White Egg........... } & .15 & .25 & .75\end{array}$

$\begin{array}{llll}\text { Forester or Seven Tops....... } & .15 & .25 & .75\end{array}$

$\begin{array}{llll}\text { Large White Globe .......... } & .15 & .25 & .75\end{array}$

Red or Purple-Top, flat

$\begin{array}{llll}\text { (strap-leaved) } \ldots \ldots \ldots \ldots \ldots \ldots & .15 & .25 & .75 \\ \text { White Top Milan ............. } & .20 & .30 & .85\end{array}$

\section{TAYLOR BROTHERS' PURE RAW} BONE MEAL, FOR LAWNS

Prices

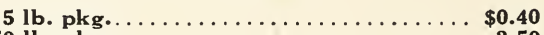

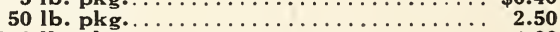
100 lb. pkg.

\section{TAYLOR BROTHERS' BUTTON BONE} Prices

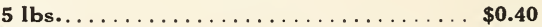

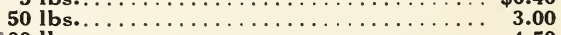

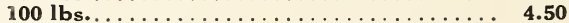

\section{BAGS}

To every order for 10 pounds and upwards, of a single item to the amount of 100 pounds, 50 cents must be added for a bag in which to ship. These bags are not returnable for credit.

\section{GARDEN HOSE}

Keystone Red, $5 / 8$ ths, coupled, ft.. . 13c Star, $5 / 8$ ths, coupled, ft........ 12c Star, $3 / 4$ in. uncoupled, per ft..... 13c Lion Concrete Hose, $1 \frac{1}{4}$ in., per ft. . 35c Gem Nozzles, $3 / 4$ in., each . . . . . . 75c

\section{DISCLAIMER}

We give no warranty, express or implied, as to description, purity, productiveness or any other matter of any seeds or bulbs we sell, and we will not be in any way responsible for them.

TAYLOR BROS. 


\section{GOLD MEDAL PLOWS}

\begin{tabular}{|c|c|c|c|c|c|}
\hline Sizes & Prices for Gold Medal Plows & $\begin{array}{l}\text { With } \\
\text { Extra } \\
\text { Share }\end{array}$ & $\begin{array}{l}\text { Share } \\
\text { and } \\
\text { Wheel }\end{array}$ & $\begin{array}{l}\text { Share } \\
\text { and } \\
\text { Jointer }\end{array}$ & $\begin{array}{c}\text { Share } \\
\text { Wheel and } \\
\text { Jointer }\end{array}$ \\
\hline $\begin{array}{l}\text { No. } 6 \\
\text { No. } 7 \\
\text { No. } 12 \\
\text { No. } 15 \\
\text { No. } 18\end{array}$ & 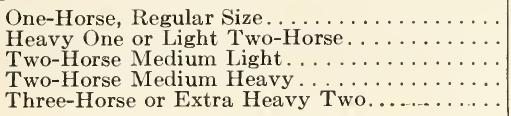 & $\begin{array}{r}\$ 9.00 \\
10.60 \\
14.76 \\
16.15 \\
20.50\end{array}$ & $\begin{array}{r}\$ 12.36 \\
16.95 \\
18.30 \\
22.60\end{array}$ & $\begin{array}{r}\$ 13.32 \\
18.54 \\
19.95 \\
24.30\end{array}$ & $\begin{array}{r}\$ 15.00 \\
21.00 \\
22.25 \\
26.40\end{array}$ \\
\hline
\end{tabular}

\section{PLANET JR.}

No. 119 Garden Plow and Cultivator, each $\$ 5.00$ No. 4 Seed Drill, each............. 18.50 No. 12 Wheel Hoe, each ............ 11.05 No, 17 Wheel Hoe, each .......... 8.00 Cultivator Handles Complete, set . . . . . . 1.90 No. 19c Plow, each............... 4.10

\section{$\mathrm{OHIO}$}

5 Tooth Cultivator Straight, Frame, Plain,

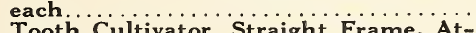
tachment, each................

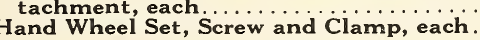

NORCROSS Out-U-Kum Weed Puller The fastest selling weed puller on the market. Pulls 'em out by the roots,

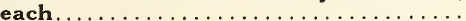

\section{SLIP POINTS}

A slip points, each.

C, D and E slip points, each.

Plain, straight, rough handles, ...............

\section{RAKES}

Cast Steel

10 teeth, heads lacquered finish, each

12 teeth, heads lacquered finish, each

14 teeth, heads lacquered finish, each

16 teeth, heads lacquered finish, each

\section{NORCROSS HAND CULTVATIORS}

5 prong, weighs 3 pounds, 4 foot handle, each...

3 prong, each

Midget, each.

\section{- MAGIC WEEDERS}

Style A.

Style H

Style G

6.00
6.50
.25

\section{TROWELS}

No. 217 A, each.

Style F

Style $\mathbf{H}$

\section{LAWN RAKES}

Wood, 20 tooth

Wire

Bamboo.

\section{GRASS HOOKS}

No. 130, Trenton

No. 100

\section{GOLD MEDAL REPAIRS \\ Standards No. 6... \$2.10 $\$$ Landsides}

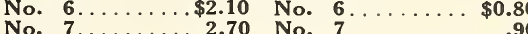

No. $12 \ldots .3 .40$ No. $12 \ldots \ldots \ldots$

No. $15 \ldots \ldots \ldots \ldots 3.50$ No. $15 \ldots \ldots \ldots \ldots$

No. $18 \ldots \ldots \ldots \ldots 4.25$ No. $18 \ldots \ldots \ldots \ldots$

Chilled mouldboards - Reversible sharesNo. $6 \ldots \ldots \ldots \ldots 2.05$ No. 6........ 60 No. $7 \ldots \ldots \ldots, 2.70$ No. $7 \ldots$

No. $12 \ldots \ldots \ldots .3 .18$ No. $12 \ldots \ldots \ldots . .70$

No. $15 \ldots \ldots \ldots, 3.80$ No. $15 \ldots . .75$

No. $18 \ldots \ldots \ldots \ldots 4.45$ No. $18 \ldots \ldots \ldots \ldots .85$

Iron Age Points, $11 / 4$ inch, No. 0 , each .....

Iron Age Points, 2 inch, No. 1, each . 20

Iron Age Points, 11/2 inch, No. 96, each .12

Iron Age Points, $21 / 2$ inch, No. 89, each... .14

Iron Age Points, 3 inch, No. 82A, each.. $\quad .16$

Iron Age Points, 31/2 inch, No. 83A, each. . $\quad .18$

Iron Age Points, 4 inch, No. 84A, each. . $\quad .19$

Iron Age Points, $4 \frac{1}{2}$ inch, No. 69, each ... . .22

Iron Age Turning Shovels, No. 16, Left and Right, each

Iron Age Sweeps, No. 11-6 inch Cultivator, Iron Age Sweeps, No. $11-8$ inch Cultivator, each....................

\section{SCYTHES}

English, 32 inch, each

Clipper, Grass, each.

Clipper, Weed, each .

Heavy Bush, each.

E. \& S. Scythes, each

\section{SNATHES}

No. 50, Light, each.

1.40

No. 105, Heavy, each.

\section{SHEARS-Hedge}

8 inch, each

...........

10 inch, each

2.65

\section{SHEARS-Grass}

No. 100, each. . . . . . . . . . . . . . .

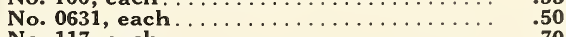

No. 117, each . . . . . . .70

No. 055E, each . . . . . . . . . . . 1.90

\section{LAWN ROLLERS \\ Diameter Length
Inches Inches
$\begin{gathered}\text { Empty } \\ \text { pounds }\end{gathered}$}

$\begin{array}{rrrrr}601 \ldots \ldots \ldots \ldots & 14 & 24 & 70 & 15.50 \\ 602 \ldots \ldots \ldots \ldots & 18 & 24 & 85 & 18.00 \\ 603 \ldots \ldots \ldots \ldots & 24 & 24 & 110 & 20.75 \\ 605 \ldots \ldots \ldots \ldots & 14 & 24 & 68 & 12.40 \\ 606 \ldots \ldots \ldots & 18 & 24 & 82 & 14.50\end{array}$

\section{PARCEL POST RATES FROM CAMDEN, N. J.}

\begin{tabular}{|c|c|c|c|}
\hline United States Parcel Post Rates & $\begin{array}{l}\text { First pound } \\
\text { or fraction }\end{array}$ & $\begin{array}{l}\text { Each addi- } \\
\text { tional pound } \\
\text { or fraction }\end{array}$ & $\begin{array}{l}\text { Packages must not exceed } \\
84 \text { inches (Length and } \\
\text { girth combined) }\end{array}$ \\
\hline $\begin{array}{l}\text { 1st Zone, within } 50 \mathrm{mi} \text {. of Camden ...... } \\
\text { 2nd Zone, over } 50 \text { and under } 150 \mathrm{mi} . \ldots \\
\text { 3rd Zone, over } 150 \text { and under } 300 \mathrm{mi} \ldots\end{array}$ & $\begin{array}{r}\$ 0.07 \\
.07 \\
.08\end{array}$ & $\begin{array}{r}\$ 0.01 \\
.01 \\
.02\end{array}$ & $\begin{array}{l}\text { Limit of weight this zone } 70 \mathrm{lbs} \text {. } \\
\text { Limit of weight this zone } 70 \mathrm{lbs} \text {. } \\
\text { Limit of weight this zone } 70 \mathrm{lbs} \text {. }\end{array}$ \\
\hline
\end{tabular}




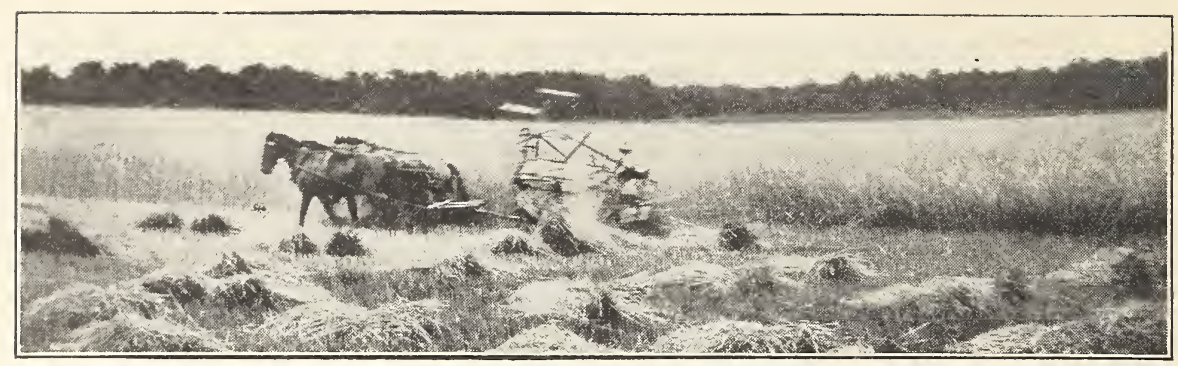

View of a 40 Acre SouthJersey Rye Field

\section{CLOVER AND FIELD SEED}

Prices Subject to Market Changes

Red Clover, Adaptable .1\% stained green $99.00 \%$ pure

Alsike Clover, Fancy..........99.00\% pure

Timothy Seed, Keystone....... 99.60\% pure $\}$ Special Prices

Crimson Clover, Fancy . .

Alfalfa Clover, Certified

Northwest

White Clover, Fancy, 60 lb...... $99.60 \%$ pure)

Sweet Clover, White, blooming, 60 lbs. per bus . . . . . . . . . .

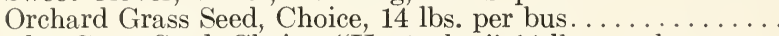

Blue Grass Seed, Choice, "Kentucky," 14 lbs. per bus. . . . .

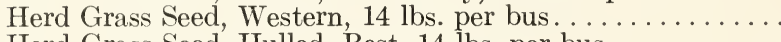

Herd Grass Seed, Hulled, Best, 14 lbs. per bus............

Millet, German, Fancy, 50 lbs. per bus. . .

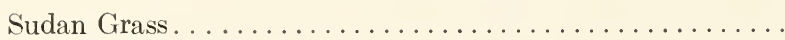

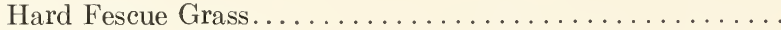

Sheep Fescue Grass. . . . . . . . . . . . . . . . . . . .

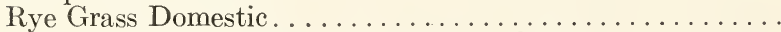

Meadow Fescue Grass or English Blue Grass............

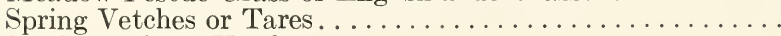

Sand or Winter Vetch.

Field Peas for Sowing, 60 lbs. per bus. . . . . . . . . . .

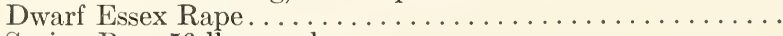

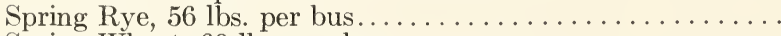

Spring Wheat, 60 lbs. per bus. . . . . . . . . . . . .

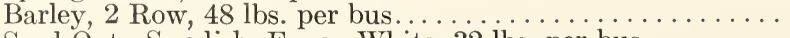

Seed Oats, Swedish, Fancy White, 32 lbs. per bus..........
$\mathrm{Lb}$

Bu. $\quad 100 \mathrm{Lb}$.

$\begin{array}{crr}\$ 0.50 & \$ 25.00 & \$ 40.00 \\ .25 & 10.00 & 15.00 \\ .32 & 3.25 & 22.00 \\ .45 & 5.00 & 35.00 \\ .25 & 2.25 & 15.00 \\ .35 & 3.50 & 25.00 \\ \text { Write for } & \text { Prices } & \\ \text { Lb. } & \text { Bu. } & \text { Cwt. } \\ \$ 0.12 & - & \$ 9.50 \\ .43 & - & 33.00 \\ .43 & - & 33.00 \\ .20 & - & 15.00 \\ .39 & - & 29.00 \\ .081 / 2 & - & 7.50 \\ .20 & - & 15.00 \\ \text { Lb. } & \text { Bu. } & \\ \$ 0.16 & \$ 3.60 & \$ 6.00 \\ .10 & - & 10.00 \\ - & 3.00 & - \\ - & 3.60 & - \\ - & - & - \\ - & 1.50 & -\end{array}$

\section{KEYSTONE VELVET LAWN GRASS SEED}

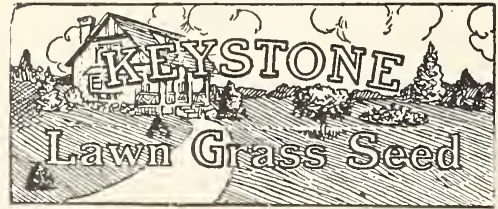

Plain

$1 / 2$ lb. pkg

$1 \mathrm{lb} . \mathrm{pkg}$

1 bu., $20 \mathrm{lbs}$
THE attractiveness of a suburban home is greatly in1 creased by a well kept, closely-cut lawn. In order to obtain this, an important feature is securing the right kind of mixture; something that will remain fresh and green in hot weather. Keystone Velvet Lawn Grass will form a deep green velvety lawn in a few weeks' time. A mixture of the finest and most desirable grasses which gives satisfactory results.

\section{With White Clover}

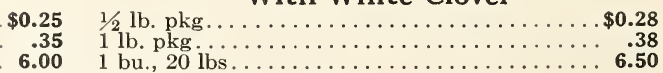

\section{SEA SHORE LAWN MIXTURE}

In order to obtain a fine lawn at the shore a foundation of ashes or clay gives the best result. With a rich top dressing and our Lawn Mixture, a fine lawn can be made in a very short time.

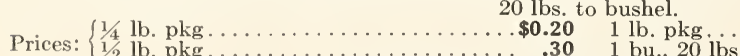

$\$ 0.50$

\section{SHADY LAWN MIXTURE}

A special mixture for all shady places, produces a fine lawn under very shady trees, where no other grass would grow.

Prices: $\left\{\begin{array}{l}1 / 4 \\ 1 / 2 \\ \text { lb. pkg }\end{array}\right.$

20 lbs. to bushel.

$\$ 0.201 \mathrm{lb}$. pkg. 
NEW RETAIL PRICES ON INSECTICIDES, 1930

\section{COATED ARSENATE}

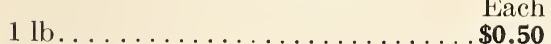

$21 / 2 \mathrm{lb} \ldots \ldots \ldots \ldots \ldots \ldots \ldots \ldots .8 \ldots$

$10 \mathrm{lb} \ldots \ldots \ldots \ldots \ldots \ldots \ldots \ldots \ldots \ldots . .25$

\section{CALCIUM ARSENATE}

$1 \mathrm{lb}$. size

Each

4 lb. size

$\$ 0.16$

5 lb. size.

.60

25 lb. size.

3.35

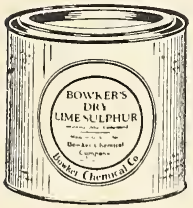

DRY LIME \& SULPHUR

1 lb. size.........\$0.25

5 lb. size.......... $\mathbf{1 . 1 5}$

$10 \mathrm{lb}$. size........ 1.90

25 lb. size.......... 2.35

$50 \mathrm{lb}$. size........ 7.20

\section{BORDEAUX MIXTURE DRY}

1 lb. size. . . . . . . . . . . . . . . . \$0.30

4 lb. size..................... 1.20

25 lb. size

6.25

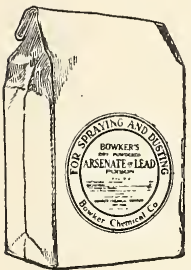

\section{ARSENATE OF} LEAD DRY

$1 \mathrm{lb}$. size........ \$0.23

4 lb. size......... $\quad .85$

5 lb. size......... 1.05

25 lb. size........ 5.15

\section{SLUG SHOTS}

5 lb. size........ \$0.55

BLUE STONE

$1 \mathrm{lb}$. size

Each

$\$ 0.12$

10 lb. size

1.10

$25 \mathrm{lb}$. size

2.50

$50 \mathrm{lb}$. size.

4.85

\section{LIQUID LIME \& SULPHUR}

Each

1 qt. size.................. \$0.35

1 gal. size. . . . . . . . . . . . . . .75

5 gal. size................ 3.15

BLACK-LEAF-40 Retail

$1 \mathrm{oz}$. bottles, each............\$0.35

1 lb. tins or bottles, each........ 1.25

$2 \mathrm{lb}$. tins, each.............. 3.25

10 lb. tins, each................ 11.85

\section{TOBACCO DUST}

$1 \mathrm{lb}$. cartons, sifter tops, per dozen. . \$0.20

In bulk, per 100 lbs. 1 per cent.

Nicotine. . . . . . . . . . . . . 3.60

In bulk, per $100 \mathrm{lbs}$. 11/2 per cent. ling wes

Nicotine.............. 4.80

\section{PEACH TREE BORER}

$1 \mathrm{lb}$. cans, each. ...........\$0.46

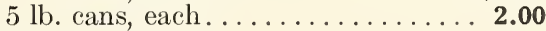

$10 \mathrm{lb}$. cans, each............. 3.60

B-K DISINFECTANT $\quad$ Each

1 qt. bottle................. 1.25

1 gal. size................ 3.00

5 gal. size................. 12.50

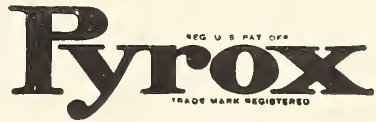

\section{Shipping Packages}

1930 Prices

$1 \mathrm{lb}$. jar, 24 to case..... $1 \mathrm{lb} . \$ \mathbf{. 5 0}$

$5 \mathrm{lb}$. drum, 12 to case... $5 \mathrm{lb} . \quad \mathbf{1 . 7 5}$

$10 \mathrm{lb}$. drum, 6 to case.... $10 \mathrm{lb} . \quad 3.00$

$25 \mathrm{lb}$. drum, 4 to case.... $25 \mathrm{lb} . \quad 6.25$

$50 \mathrm{lb}$ drum........... $50 \mathrm{lb} .10 .75$

$100 \mathrm{lb}$ drum ............. $100 \mathrm{lb} . \quad \mathbf{1 5 . 5 0}$

$300 \mathrm{lb}$. barrel............ $300 \mathrm{lb.} \quad \mathbf{4 3 . 5 0}$

\section{JERSEY GALVANIZED POULTRY WIRE NETTING}

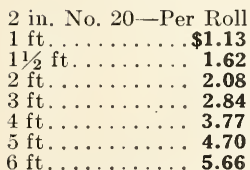

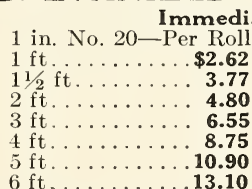

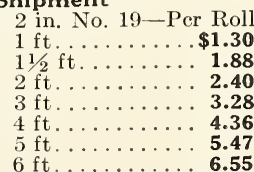

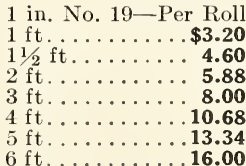

Roll contains 150 running feet. All other sizes of Wire and Mesh quoted on application F. O. B. Car Camden.

\section{Our Terms are Strictly Cash with the Order}

We do not send C. O. D., as the cost of collecting return charges is quite an unnecessary item of expcuse and the prices being given, we can conceive of no necessity, to warrant goods being so sent.

\section{How to Send Money}

Remittances may be made at our risk by any of the following methods, viz.: Postal Money Order, or Express Company's Money Order.

The rates charged for Postal Money Orders and Express Money Orders are now so low that these are the best ways to remit. We will bear the expense of sending money in either of these ways. Deduct the cost of the order from amount sent.

When Money Orders cannot be obtained, letters containing money should always be registered. Money in ordinary letters is unsafe. If currency is sent by express, the charges should be prepaid.

\section{Personal Checks}

If personal checks are used they should be certified, otherwise shipment of your order may be delayed pcriding collection.

Name and Address Should Always Be Given

Frequently we receive unsigned letters. Sometimes they contain money and orders. Sometimes too letters are received in which the name of the town is left out and the postmark is blurred. We cannot fill orders unless we know the name and address of the buyer. 


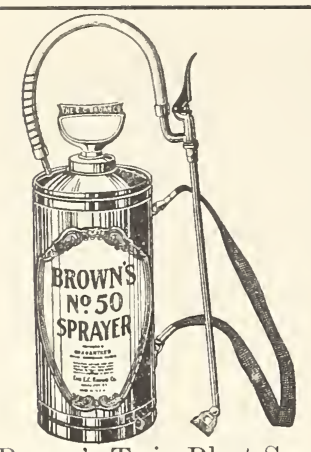

Brown's Twin Blast Sprayer. Brown's Whirlwind Sprayer Brown's 30D Sprayer. Brown's 201D Sprayer. .

\section{BROWN SPRAYERS}

No. 9 AUTO SPRAY. 4 gallon capacity, extra strong tank. Galvanized, \$6.95 each. Brass tank, $\$ 10.50$ each.

No. 50D AUTO SPRAY. $21 / 2$ gallon capacity, $\$ 6.00$ each.

No. 6 AUTO BUCKET SPRAY. Very powerful, 200 lb. pressure. Brass $\$ 4.75$ each.

No. 26C AUTO SPRAY. No other machine like it for power. Continuous atomizer, price $\$ 1.60$ each.

No. 23C AUTO SPRAY ATOMIZER. For spraying cattle. Price $\$ 3.25$ each.

Brown Sprayers Are the Most Complete Line on the Market Each $\$ 0.75$

.50

15.25

6.10

\section{LAWN SPRINKLERS}

Brown's Whirling Showers. . . $\$ 2.75$

Brown's Revolo.................... 1.75

Brown's Ready Showers.......... 1.00

\section{FELKERS FOUNTAINS \& FEEDERS}

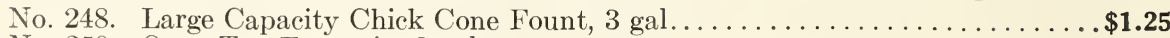

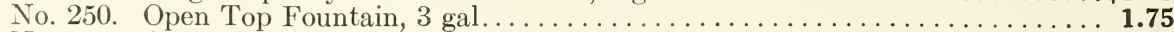

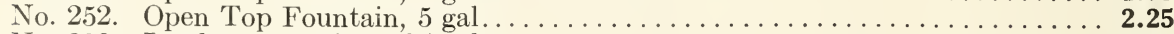

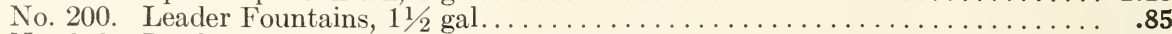

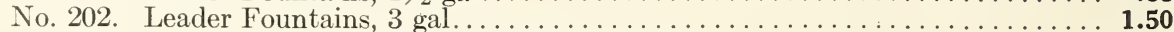

No. 226. Round Table Drinking Fountains, 5 gal..................

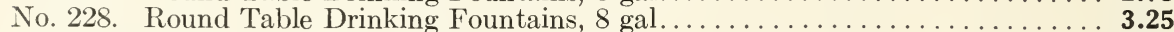

Giant Mash Feeder, capacity over 100 lbs., each ....................... 10.00

No. 318. Sr. Chicken Feeder, $30^{\prime \prime}$ long, holds 25 lbs................... 2.50

No. 010. Jar Founts, fit mason screw top jars, each.....................

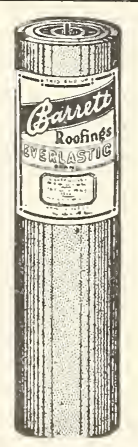

\section{BARRETT ROOFINGS}

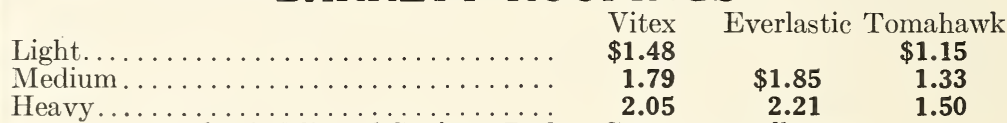

Everlastic Mineral Surface, Red or Green, per roll, \$2.08

\section{MINERAL SURFACED SHINGLES}

(Red, Green or Blue Black) Weight Size

10-in. Multi Shingles, 4 in.

Exposure............. Square 190 lbs. sq. $10 \quad x 36$ in. $\quad \$ 5.22$ sq.

Octagonal Strip Shingles, 4 in.

Exposure................ Square 190 lbs. sq. $11 \times 33 \frac{5}{8}$ in. $\quad 4.97$ sq. Hexagonal Strip Shingles... Square 160 lbs. sq. $11 \frac{11}{32} \times 36$ in. $\quad \mathbf{4 . 7 4}$ sq.

\section{LAWN MOWERS}

MIDDY LAWN MOWERS

12 in., each................\$6.50

14 in., each................. 6.75

16 in., each............... 7.00

\section{COLONIAL LAWN MOWERS}

12 in., Ball Bearing..............\$8.65

14 in., Ball Bearing............ 9.10

16 in., Ball Bearing.............. 9.50

18 in., Ball Bearing............ 9.90

LAKEWOOD LAWN MOWERS

14 in., Ball Bearing, each.......\$10.10

16 in., Ball Bearing, each. . . . . . 10.50

18 in., Ball Bearing, each. . . . . . . . 11.00

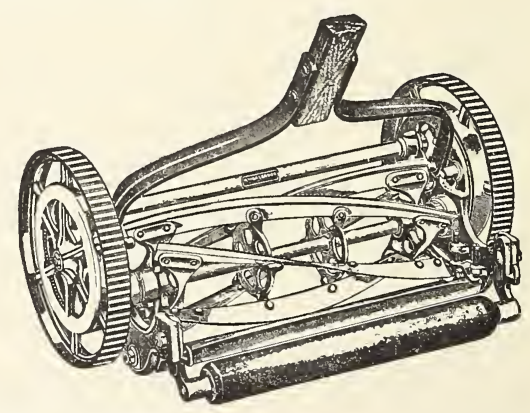

AMBASSADOR LAWN MOWER

16 inch cut, each ...........\$12.65 18 inch cut, each...........\$13.50

\section{TAYLOR BROS. \\ CAMDEN, ${ }_{1}$ NEW JERSEY}





\section{O. D. 0 . \\ BUTTERMILK BABY CHICK STARTING FOOD}

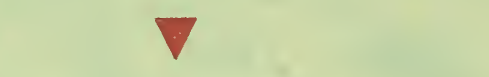

Scientific Analysis:

O. D. 0 .

Protein, 11.25 per cent. Fat, 3.40 per cent.

Carbohydrate, 67 per cent. Nitrogen,

Free Extract, 64 per cent. Crude

Fibre, 3.70 per cent.

Prices:

5 pound packages $\$ 0.45$ each

10 pound packages .80 each

25 pound bags 1.95 each

50 pound bags 2.80 each

100 pound bags

5.00 each

\section{Keystone and Dandy}

Poultry, Pigeon and Chick Foods 